\title{
Institutionalizing HCI in Asia
}

\author{
Andy Smith ${ }^{1}$, Anirudha Joshi ${ }^{2}$, Zhengjie $\mathrm{Liu}^{3}$, Liam Bannon ${ }^{4}$, \\ Jan Gulliksen ${ }^{5}$, and Christina $\mathrm{Li}^{1}$ \\ ${ }^{1}$ Thames Valley University United Kingdom \\ andy.smith@tvu.ac.uk \\ ${ }^{2}$ Industrial Design Centre, IIT Bombay, Mumbai, India \\ anirudha@ittb.ac.in \\ ${ }^{3}$ Sino-European Usability Center, Dalian Maritime University \\ liuzhj@dlmu.edu.cn \\ ${ }^{4}$ Interaction Design Centre, University of Limerick, Ireland \\ liam.bannon@ul.ie \\ ${ }^{5}$ Uppsala University, Sweden \\ jan.gulliksen@it.uu.se
}

\begin{abstract}
In this paper we investigate the problems and potential solutions to the effective establishment of HCI and usability in India and China. Our discussion is motivated by five years of collaboration with relevant bodies in both countries through EU-funded projects encouraging the development of a usability culture in academic and industrial sectors. In order to contribute to socially-responsible interaction in these countries the 'institutionalization' of $\mathrm{HCI}$ is necessary. For us, this involves three elements: firstly an appropriation of HCI concepts and methods to suit the local country / culture, secondly the forming of a national organization around the reshaped discipline that can actively promote HCI in industry and academia and establish links with local national organizations, and thirdly the roll-out of effective usability practice in industry. Some efforts made in this regard are briefly outlined.
\end{abstract}

Keywords: cross-cultural usability, India, China, institutionalization.

\section{Introduction}

With a combined population accounting for nearly two-fifths of humanity, the neighbouring Asian countries of India and China, are two of the world's fastestgrowing economies. These countries have embarked on radical, liberalising economic reforms that are resulting in improved living standards for many, though not all, its peoples. At the same time, there are huge problems in terms of providing for adequate housing, sanitation, education and welfare for all the population. In India more than half of women and 30 per cent of men remain illiterate. In China the economic inequalities between Western (mainly agricultural and Eastern (highly industrialised) China is resulting in migration from poverty-stricken rural areas to the fastly developing cities.

Nowhere is the so-called "digital divide" - in terms of those who have, or have not, access to computing and communication services and infrastructure - more 
pronounced than in some Asian countries. Internet penetration ranges from below $1 \%$ in countries like Bangladesh, Cambodia and Laos, to above $65 \%$ in South Korea. Furthermore, both India and China have their own internal digital divides. Whilst regions such as Shanghai and Bangalore have almost the same level of Internet and mobile phone connections as Western nations there are rural areas across India and in Western China which are at the other end of the spectrum.

Both India and China have fast developing IT industries supporting both local and off-shoring software development. However, again, the internal divides within the Indian and Chinese economies are evident. In India whilst at US\$30bn the IT industry contributed 4.1 per cent of GDP in 2005 it employed only $0.25 \%$ of the entire workforce. Similarly, although China is the world's fourth-richest economy, it has a per capita GDP of only US $\$ 17,000$ compared to the OECD average of US $\$ 30,000$. These facts are evident in the Economist's 'e-readiness rankings' [1] where in 2006 India and China ranked $53^{\text {rd }}$ and $57^{\text {th }}$ out of 58 countries.

There are signs of a new world economic and political order, where countries like India, Brazil and China are emerging key players. There are signs of an awareness of their increased economic and political "muscle", especially as they begin to develop bilateral and multilateral agreements among themselves, increasing their strategic importance on the world stage. India and China could push globalization in a new direction, ending 600 years of Western dominance [2]. Significantly as part of an agreed programme the countries have called for the combination of Indian software technology with Chinese hardware technology to achieve world leadership in the global information technology industry.

It is against these generic economic and specific ICT backgrounds that we seek to explore the role that a human-centred approach to the development and use of ICT could play in the socially-responsible development of the Indian and Chinese information societies. The human-centred approach that we refer to here is of key importance in major segments of the ICT industry, in software applications for business and industry, in consumer markets, and in areas of health and defence. ${ }^{1}$ Successful growth of the ICT sectors in these countries, focusing on both internal and external markets, will be dependent on paying increased attention to human, social and cultural factors.

Given that the disciplines of HCI and usability can be traced back over two decades or more in certain Western countries, both India and China are at a relatively early stage of development in this regard, having paid very little attention to the discipline of human-computer interaction in Universities, nor to a formal implementation of usability principles as understood internationally in industrial practice. The reasons for this are diverse, but are rooted in both countries highly skilled, yet technically oriented, approach to computing as a subject and software development as a practice, and in the "backstage" software maintenance work originally being outsourced. In addition, the HCI area in China has been slow to develop due to a socio-political perspective, that had been suspicious of disciplines

${ }^{1}$ In what follows we will use the generic term HCI (Human-Computer Interaction) for this human-centred approach, encompassing a range of human-technology interactions - usability, interaction design, user-centred design, participative design, collaborative working, etc. 
such as psychology and sociology - disciplines that underpin much of the HCI approach.

According to Rogers [4] the first two of five stages in the diffusion of innovation are knowledge (learning about the existence and function of the innovation) and persuasion (becoming convinced of the value of the innovation). Through European Union funded projects we have attempted to assist in developing knowledge about HCI and encouraging individuals and organisations to implement such knowledge. The Indo European Systems Usability Partnership (IESUP) [5] and the Sino European Systems Usability Network (SESUN) [www.sesun-usability.org] have enabled collaboration between academics and practitioners in India and China with their counterparts in Europe and globally. We have worked in six major cities across India and eight cities in China with the aim of raising the profile of HCI at institutional levels in both academia and industry. This paper reports on some of our experiences on these projects. Specifically, in the next sections, we discuss

- the state of play of HCI and usability in India and China,

- differences in HCI in the these countries compared to the West,

- a model for the institutionalization of HCI.

\section{Usability Practice in Indian and Chinese industry}

Before exploring the development of HCI and usability it will be useful to provide a framework for analysis. The Usability Maturity Model (UMM) [6] provides us one possible measure of the progress of HCI capabilities in industry. This describes the maturity of the company in terms of various levels and gives indicators for each level. Table 1 summarizes the levels of the UMM and their indicators.

Table 1. Levels of usability maturity and their indicators

\begin{tabular}{|l|l|}
\hline Level & Indicators \\
\hline Unrecognized & (no indicators) \\
\hline Recognised & Problem recognition, performed processes \\
\hline Considered & Quality in use awareness, user focus \\
\hline Implemented & User involvement, human factors technology, human factors skills \\
\hline Integrated & Integration, improvement, iteration \\
\hline Institutionalized & Human-centred leadership, organizational human-centeredness \\
\hline
\end{tabular}

The darker lines under Unrecognized and Implemented indicate current barriers in industry.

A company is considered in the unrecognized level if most people in the company believe that there are no usability problems in its products and investments and developing HCI skills are not warranted. A progression to the recognized level is typically unsystematic. This level is marked with possibly sincere, but haphazard 
attempts to resolve the usability issues of the problem cases. A company moves to the considered level when it starts making systematic financial investments. This happens either in terms of hiring $\mathrm{HCI}$ consultants on specific projects or by inviting professionals to conduct training. A company moves to the implemented level when it realizes that it needs to use HCI skills on an on-going basis and sets up a specialized HCI group. At this level, the company has the capability of producing usable products, but it does not use this capability consistently in all projects. A company becomes integrated when its HCI activity becomes mainstream and routine for all projects, not just for critical projects. A company would be considered institutionalized when it starts considering itself as a human-centred solutions company rather than a technology company.

\subsection{India}

The emergence of HCI in India occurred in the early 1990s [3]. However by the mid nineties the only Indian software companies that were employing professional user interface designers were multimedia content developers which could not be considered to be in the 'mainstream' of software development. A few large software services companies offered a course on graphical user interfaces in their training schedule, but the quality of these courses was often poor. HCI was rarely taken seriously or applied in projects.

The dot-com boom attracted a few talented and largely self-taught professionals into the HCI fold. In the second half of the nineties, many of the early multimedia companies had evolved into mature web and e-learning operations with active interface design and information architecture groups. Some of these would have been at the implemented level of UMM, while others at the considered level. Around this time, international companies set up software development centres in India. Some of these also set up usability groups, consistent with their organizational structures elsewhere. Many of these companies would be at the implemented level of UMM.

The dot-com bust towards the end of the nineties brought many young, creative professionals into the software service industry. Also a few medium-sized software service companies in India began making investments in HCI. They recognized that the web was increasingly becoming an important medium of software delivery, and delivery needed significant design inputs. For an estimated 10\% of Indian software companies, this was the transition from recognized to considered. But most of the Indian software industry was at the unrecognized level till the end of the nineties.

The first seven years of the new millennium has seen a significant transition in terms of acceptability of HCI in India in industry, community and academia. The few software services companies that started out early are today 'on the verge' of the integrated level of the UMM. The HCI practice within these organizations is rapidly becoming mainstream and some process improvement is already visible. Also in this period, we have witnessed a couple of international companies offering services in HCI through their Indian operations. Meanwhile, a few of the larger software services companies have moved from unrecognized level to the considered and implemented levels. 


\subsection{China}

HCI and usability emerged in China even later than it did in India, really only establishing itself as a field after 2000 and especially more significantly since 2003 . The reasons for this late development are as a result of a variety of social, economic and cultural reasons. Firstly historic levels of Chinese economic and industrial development have not been conducive to HCI. From the 1950s to 1980s, China had a planned economy. At this time disciplines like psychology and sociology suffered from various restrictions before and during the Cultural Revolution. There has long been a preference for technology-related disciplines rather than humanities-related disciplines in Chinese society.

However with the rapid growth of the Chinese economy and the process of globalization in recent years, Chinese enterprises realized that they had to strengthen their competitive edge to be able to survive and compete in the future. At the same time, more and more multinational companies have entered the Chinese market. These two factors have brought about a rapid increase in demand for usability. Indeed usability practice in China started from activities conducted by multinational companies, some setting up usability groups. Stiff international competition and the desire for development have also made user experience an important issue for many leading Chinese companies. Some maintain usability groups of over twenty people and have integrated user-centered design (UCD) into their processes.

In order to better understand the current situation of usability in China, an organizational human-centeredness assessment at Chinese IT enterprises was undertaken [7] using the usability maturity model. Although the study was based on a small sample the assessment results showed that the usability maturity level for all enterprises was only at the recognized level. Because the enterprises represent the advanced level in the Chinese IT sector, this evaluation actually reflected the current maturity level of most leading enterprises in the sector. As part of the current SinoEuropean Systems Usability Network project further usability maturity studies are currently being undertaken.

Although the number of people in China dedicated full-time to usability practice is still small, maybe around 400, many product designers and developers are interested in usability. They are young, full of enthusiasm, and eager to learn. Of the people who are most interested in usability, quite a few are from design backgrounds, probably because many companies employ design-trained people for user-interface design jobs.

At the first Sino-European Usability Seminar Tour held in Beijing, Shanghai, and Shenzhen, in 2005 more than 200 people attended, with eighty percent from industry. Several companies sent more than ten of their employees to the event. A survey we conducted during the tour revealed that most of these companies have set up usabilityrelated positions and departments. The respondents said they believed that usability would become more important in their organizations and that the major challenges at the moment are to master usability practices and skills and then to get their work recognized by their bosses and product-line units. Therefore, they wanted to attend training courses and learn from case studies so as to be able to start practicing usability in their daily work quickly. 


\subsection{The Problems of Off-Shoring}

In both India and China (but most particularly in India) in the past two decades, the IT industry has relied on providing quality software services in a cost-effective manner. It has effectively leveraged the huge difference between the labour costs of equivalent skills in India and the developed part of the world. IT companies have developed excellent software engineering processes to manage such projects effectively. For example, the largest number of ' $\mathrm{CMM}$ level 5' companies is in India [8].

In the early days, much of the requirements and the initial design specifications were all done in the West, and then "shipped" overseas for the actual development and implementation. From the perspective of designing human-computer interaction, this had significant negative consequences. This effectively transfers many HCI and usability issues in the first part of the project to the client. At the end of the development life cycle products are evaluated for quality against requirements and sent back for 'acceptance testing' to the client. Formal usability evaluations were rarely done until recently. Informal usability evaluations, if at all, were usually carried out as part of acceptance tests and were managed as 'upgrades' or 'change requests' as they were deviations from the original requirements. Having high CMM levels did not help as such certifications are largely based on software engineering literature. Traditional software engineering process literature does not integrate $\mathrm{HCI}$ activities well into the software engineering processes [9].

In effect the prevalence of this kind of off-shoring project has led to the marginalization of HCI skills in India and China in the early days of out-sourcing. This led to the image that India and China are mainly destinations of off-shoring 'low-end', implementation oriented software work. More recently, though, these very skills are being considered as important means by the IT companies to move up the 'value chain' and provide complete, full-lifecycle solutions. Usability groups in some large IT services companies are currently flooded with work and scaling up rapidly from 10-20 people a few years ago to 100-300 people now, though 'full-lifecycle' projects are still relatively few.

\section{Building Education, Research and Development}

Education in HCI has begun only recently in India. Before 2000 the Industrial Design Centre of the Indian Institute of Technology, Bombay started two formal courses related to interaction design, but it was, to our knowledge, the only university in India doing so at that time. Since 2000 some other universities (National Institute of Design, and Department of Design, IIT Guwahati) have become active in this field, but the overall coverage is still poor. Interestingly, the interest in $\mathrm{HCI}$ is present in the design schools in India, rather than in the computing or technology sections of the more well-known Indian Institutes of Technology, or in psychology or human sciences.

The HCI community of practitioners in India has, however, become active in the last five years. A mailing list of Indian HCI professionals was formed in the year 2001 [http://groups.yahoo.com/group/hciidc/]. The membership of this group has since grown from about 200 at its inception to over 1100 today. Other special interest 
mailing lists and city-level groups have since been formed. The South India chapter of the Association for Computing Machinery - Special Interest Group on Computer Human Interaction (ACM SIGCHI) was formed in 2001. Since the year 2001, the chapter organizes Easy - the first annual conference related to HCI in India.

The last few years have seen new activities emerge. Our Indo-European Systems Usability Partnership allowed for the organization of a series of seminars and culminated in the first peer-reviewed conference on HCI in India - the all-India Conference on HCI (IHCI 2004) held in Bangalore. There are currently plans to form additional ACM SIGCHI chapters, a chapter of the Usability Professionals Association (UPA) and possibly a national body of professionals.

Regarding China, although some interest in HCI can be traced back in certain universities to the 1990s, as with India, it is only in the last few years that it has begun to take root. Today there are about twenty institutions working in HCI, including computer science, industrial engineering, and psychology departments in universities, research institutes, and industrial $\mathrm{R}+\mathrm{D}$ departments. Although there are over a thousand computer-science departments in Chinese universities, only ten offer $\mathrm{HCI}$ courses to undergraduate students. Where they do exist the majority of HCI people come from either computer science or design backgrounds rather than psychology or other disciplines. Reflecting the historical technological expertise in China, HCI research in computing / IT centres is still largely concentrated on HCI technologies like multimodal interfaces using speech and pen, and emotion-based interaction while paying less attention to industry's needs in relation to user-centred design.

However there has been significant growth in the usability field in China, and interest groups have become involved in the formation of professional organizations. Founded in 2003 ChinaUI [http://www.chinaui.com] is China's most popular user interface design and usability website with some 85,000 registered members nationwide. Founded in 2004, ACM SIGCHI China [http://www.hci.org.cn] sponsors an annual national conference. UPA China [http://www.upachna.org] was set up in 2004 in Shanghai and organizes the User Friendly conference every year. The large HCI International Conference for 2007 is taking place in China this year.

\section{Cultural Differences}

It is clear from the analysis to date that the development of usability and HCI in India and China would appear to have much in common. Both countries are experiencing rapid growth from a very low base of only five or ten years ago. The progress of the disciplines is of great interest to Western experts, particularly so given the clear cultural and business environment differences both between these countries and Western ones.

Significant cultural differences also exist within each country. China's population consists of 56 officially recognized nationalities, with the Han nationality (94\%) most numerous. Although there are many different local dialects and accents, Chinese writing is uniform throughout the country, owing to the government's long-standing efforts to unify the people through a uniform language. Although India is bound as a nation as a result of its common history, the differing regions, 28 states and 7 union territories have their own distinct cultural identities. In contrast to China, India has 18 
official languages and about 300 dialects. Although English is widely spoken, it is not an official language.

The global HCI community's understanding of the practical relevance of cultural issues in HCI has mirrored the timescale of the development of the subjects in India and China. In the last ten years HCI practitioners have changed their approach significantly to embrace cross-cultural development. When defining culture, researchers often refer to patterns of values, attitudes, and behaviours which are shared by two or more people. They further point out that culture is socially acquired, and that relationships with other people, relationships with the environment, and assumptions in term of space and language (for example) affect and shape culture, and are themselves affected by the culture [10]. Culture remains difficult to study, alone and certainly in relation to HCI practices. It is in particularly difficult to identify meanings, attitudes and expectations, not to mention the deeply embedded values and beliefs behind people's thoughts, behaviours and actions. People's behaviours might be influenced by other factors (e.g., environmental conditions) rather than by their cultural traits, and the reasons for, and meaning of, an action can seldom be observed wholly and directly. We would also note that we must be careful in ascribing people's specific behaviours as being due to national "cultural" differences, as nation states themselves are, in a sense, recent fabrications, and thus their citizens are not homogeneous, and stereotyping of people from different countries can often result, without a proper scientific foundation.

Studies in cross-cultural HCI have often embraced some consideration of cultural cognitive models. Cultural models are based on the assumption that cultural differences are the result of social learning and are of very long duration. These cultural models serve as a point of departure as described by Hoft [11] for example Edward Hall [12], Trompenaars [13], Victor [14], and Hofstede [15]. The model of national culture proposed by Hofstede has been frequently involved in the intercultural study of the use of systems [16]. However being based on a study of IBM employees in the 1970s there are significant gaps for the developing world, not to mention the fact that there is increasing critique of both the methods used by Hofstede and the fundamental cultural "dimensions" he has put forward [17].

Most Western software developers would support the principles of user-centred design [18] but underlying concepts and assumptions are derived from USA / Northern European cultures. It is inevitable that those tools and techniques which involve users the most would be those very techniques which were most sensitive to cultural issues. Up to now, both non-native and native personnel have practiced usability in India and China. However, with the growth of local usability expertise, the "localization" of usability practice is a necessary and inevitable trend.

\section{Institutionalizing HCI}

In both India and China the number of usability professional is growing significantly. Through a wide range of engagements with usability practitioners it is clear that there is a considerable appetite to learn about Western HCI case studies in the expectation that these can be implemented locally. However there are two problems to overcome: 
a) Firstly a richer, more nuanced, understanding of HCI is necessary in order for the most effective tools or techniques to be successfully selected and implemented this implies a much broader 'education in HCI' rather than just 'training in tools'. We need to be able to judge the appropriateness of particular tools for particular tasks, and this requires HCI education, not simply training in techniques.

b) Secondly the cultural and organisational differences between countries means that HCI tools and techniques that have been developed in Western countries may not be effective in developing countries. What is required is the localisation of methods to meet local requirements.

We seek therefore to support the institutionalisation of HCI in the two separate countries, as one key way to ensure that HCI is properly developed and implemented. Institutionalisation needs to exist both in academia (so that effective teaching and research can be supported) and industry (so that software engineers can understand and implement the principles and practices of $\mathrm{HCI}$ ).

We see this institutionalisation to have three elements; firstly the reconceptualization / redefinition of HCI in the local country or culture, secondly the embedding of HCI concerns and its importance in local national organizations and finally the rollout of usability training and best practice into industry. We recognize that in practice these elements will occur in parallel (and indeed all have started) but the critical issue is to ensure sufficient feedback between the elements.

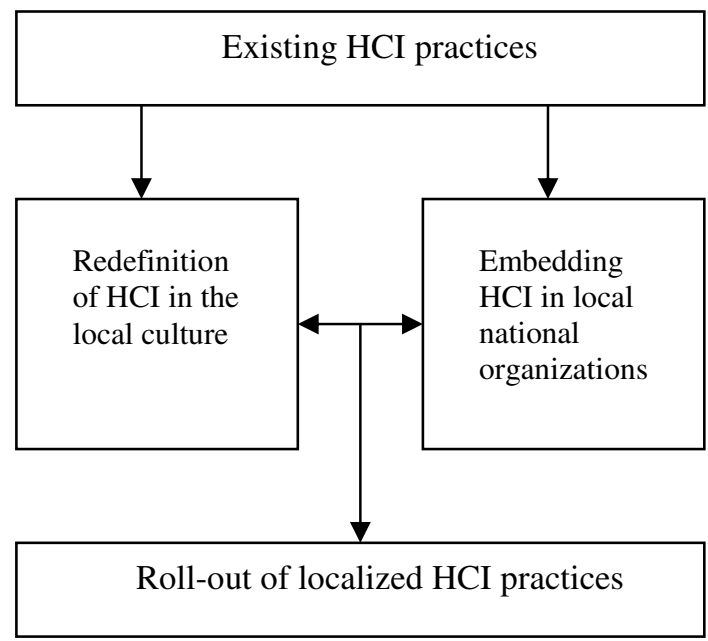

Fig. 1. Elements in the institutionalization of $\mathrm{HCI}$ in India and China

\section{Redefining HCI in the Local Setting}

For HCI and usability to be useful and acceptable in India and China they need to adapt to the needs of the local societies, both to individuals who interact with the artefacts that are produced, and with the development communities who produce them. Of course this redefinition does not need to start from scratch, but neither is it 
appropriate to apply methods that may have been successful elsewhere without local testing for suitability. In order to achieve this redefinition both indigenous and global approaches are required, critically involving collaboration between them.

\subsection{Localizing Established HCI Methods}

In the process of developing local expertise, it is, of course, necessary for local experts to learn from the experiences accumulated over the past twenty years in the West. Nevertheless, there has long been a discussion as to whether the usability methods developed in the West can be suitably used in other cultures.

User based testing / evaluation techniques are often based upon the concepts of cooperative evaluation or contextual inquiry [19] and are embodied in a variety of methods such as thinking-aloud protocols. The methods aim to gain meaningful information about the user's work by empowering the users in direct conversation with the designer on equal terms. These 'traditional' methods of testing are difficult and costly to operate across cultures and remote geographical locations [20]. As an illustration Murphy [21] describes the problems that can arise in international usability testing. Usability professionals trying to undertake such techniques in Asian cultures often find that users have particular difficulty [22]. Users vary in their ability and willingness to articulate their thoughts to the evaluator depending on both their individual personality and cultural background. Recognizing that in many Asian countries that the main challenge with usability testing is that it is impolite to tell someone they have a bad design, Chavan [24] has developed the "Bollywood" method (which inherits from the Bollywood film genre, which typically involves 'emotionally involved plots with great dramatic flourish) to reduce user inhibitions.

Based on our experiences, the fundamental principles of HCI and usability engineering undoubtedly work well, however, the operational details, for example, participant recruitment and scheduling, the use of informed consent agreements, and manners and behavior when interacting with participants, need to be adjusted for the local culture. In our own studies within IESUP we have found evidence that Indian users have significant difficulty in engaging in role-playing situations as is required in think-aloud usability methods. Furthermore we have found some evidence that Indian users do not adapt readily to sequential task-based testing. This is in accordance with India's supposed polycronic culture as defined in cultural models [12] in which multiple tasks are handled at the same time, and time is subordinate to interpersonal relations. However more work is required to recommend any generalisable guidelines.

Even so, these issues require us to consider the degree in which the replication of Western approaches to HCI in India and China is to be encouraged. The problem is particularly acute as our evidence in practical projects in India and China is that the developing local usability communities are probably too keen to implement 'best practice'; from the West before fully testing its relevance in the local culture.

\subsection{Building HCI into Off-Shoring Development}

Current techniques in user-centred design extensively rely on close and continuous contact of the design team with the users. In the context of the model of global software development discussed earlier, the business model of many software 
companies relies on distributed development, with the bulk of the work being done off-shore. The people involved in the on-site components of projects typically are involved in marketing or project management activities and are often disinclined to do HCI related tasks.

Whilst the dominance of off-shoring / outsourcing have significantly constrained the development of HCI in India, and to a lesser extent in China, it does lead to a significant research and development issue. How do we integrate sound user-centred systems design (UCSD) principles into an outsourcing development approach where the end-user is remote from the development team? Asian software companies are very process driven in their software engineering processes. However, current $\mathrm{HCI}$ techniques have not been well-integrated in software engineering [9]. There are both old and new proposals to integrate HCI in traditional software engineering process models such as the waterfall model, but these are nascent proposals that have not yet been widely verified and accepted. Also there are several other process models such as the Rational Unified Process or the wide variety of agile process models where more such work is necessary. There have been a few experiments at training business analysts in HCI techniques or hiring local usability resources. More experimentation and experience sharing is required, particularly in the area of integration between HCI, software engineering and business analysis.

As part of the re-definition of HCI, localized methods for 'remote UCSD' could enhance both the range and quality of off-shoring IT services. Specifically researchers in India and China need to collaborate with colleagues in the West to address the following issues:

- How to develop systems with an active user focus when the users are thousands of miles away?

- How best to organize and distribute usability expertise between the range of personnel involved?

- How can cultural differences between users and developers be best handled?

\section{Embedding HCI in Local National Organizations}

The institutionalisation of HCI includes developing institutional structures for the ownership, indigenous development and promotion of the discipline. We believe it is critical for developing countries to take local ownership of the discipline. However helpful international groups such as $\mathrm{CHI}$ chapters can be, we believe that national organisations are critical to ensure effective HCI development in promoting the localisation of methods, supporting the growth of HCI teaching (and research) in universities and disseminating best practice within industry (most notably in organisations where HCI and usability has yet to take root).

IESUP has enabled collaboration with the Computer Society of India (CSI) and SESUN is currently establishing active collaboration with the CSIP (Chinese Ministry of Information Industry - Software and Integrated Circuit Promotion Centre). This has helped spread awareness, but it is important that independent national organizations with linkages to established organizations be formed (on the lines of the British HCI Group a Specialist Group of the British Computer Society). In India few HCI practitioners are members of the Computer Society of India in the first place, and 
even if all of them were to enrol, they are most likely to be lost in a larger organization. Through current contact and future activities we hope to be able to provide whatever support we can to these organisations (and others) to guide the socially responsible development of $\mathrm{HCI}$ in their respective countries.

Formal education, or the lack of it, is an important reason why there is so little mainstream awareness about HCI. Even today, very few undergraduate students have the option of taking HCI courses. More courses and programmes along with the 'pull' from the industry are needed to make a difference in the area of education. The field of HCI has widely adapted many techniques from several disciplines. There are techniques for understanding users and analyzing their needs, as well as techniques for design and iterative usability evaluation. HCI skills need to be learned systematically before they can be used reliably and confidently. Estimates of the number of HCI professionals needed in India vary from a modest 40,000 to a high of 400,000 within the next few years. But even the smaller figure is much higher than the currently estimated community strength of about 2,200 professionals in the country.

\section{Roll Out of Localized Usability Practice}

HCI activities will become sustainable only if industry is able to convert the skills into profitable revenue. Arguably, if HCI skills could push industry up the value chain, currently projected levels of IT growth could potentially be greater. But this potential does not get realized in the bottom line unless entrepreneurship can harness it well.

Training a sufficient number of well qualified HCI and usability personnel could well be the biggest challenge and the hardest to achieve. There is a need to increase the number of trained people as well as the need to develop new, integrated techniques that work well with few HCI professionals and much support from other disciplines. Usability engineering needs a multidisciplinary team, especially personnel with psychology and HCI design backgrounds. There is a severe lack of such personnel in both Indian and Chinese industry; almost all the employees in the development departments are trained in computer science or related technology oriented specialties.

Training courses for user interface design and usability as well as corresponding certifications should be provided to show the value to the profession and to encourage enterprises to set up specific job positions for usability experts. This effort would foster the accumulation and development of related expertise in the industry.

Pilot projects need to be carefully selected and conducted to provide successful cases for convincing more enterprises to adopt UCD approaches. Emphasis of the pilot should be placed on the localization of the UCD methods (especially their suitability for technology-oriented staff), cost-benefit analysis, as well as development of guidelines for the methods, training materials, case studies, and tools.

International or industrial standards, like ISO 9000, CMM etc., have been well respected in both the Indian and Chinese IT sector. Evaluations and certifications for 
product usability and process usability maturity might be a potential factor that can substantially drive the acceptance of usability engineering in industry.

In the path to usability maturity, and given the current context in both India and China, two changes in usability maturity model levels will be the hardest to achieve:

From Unrecognized to Recognized: Firstly moving from the unrecognized level to the recognized level represents a major cultural change for a technology company. In the past, technology has been a major strength of the company and was always sufficient to deliver what a client asked for. However, the very success of technology and its wide-spread use has changed the world. This change is not necessarily obvious to an 'industry-insider'. It is important to consciously recognize that success of the past is the very reason why the future needs to be different.

From Implemented to Integrated: Once a company recognizes that they have a problem, they can usually figure out how to solve it. It makes investments (on training or consultants) and moves to considered. If it can see the value, it sets us a specialized HCI group and moves to implemented. This is where they run into the next major barrier. Moving to from implemented to integrated requires a dual challenge. Firstly, it is a significant change of scale - it is not the relatively simple matter of hiring a small group of people any more. HCI skills need to be a part of each project. Estimates of the amount of HCI effort required in a mature operation vary from $5 \%$ to $15 \%$. That can be quite a task for a company with 5,000+ people and 500+ projects annually. Secondly, being integrated means investments into ongoing process improvement, something which will strain already limited HCI workforce

\section{The Future}

Whichever way one looks at it, it seems that HCI will have a more significant role to play in the next few years in the Indian and Chinese software industries. Even the Chinese government in the recently published National Science and Technology Plan for 2006-2020 for the first time uses phrases like human-centered and ease of use.

The impact of HCI on industry itself could be dramatic. The Indian software services industry in particular has already moved on from the perception of being 'cheap, outsourcing destination of largely low-end work' to being 'providers of solutions and services in a highly process-driven, professional environment'. HCI inputs can help the industry move ahead in this course as 'providers of innovative, proactive, full-lifecycle, integrated solutions'.

Supported by enhanced HCI and usability practices the sustained growth of the IT sector in both India and China will contribute to overall economic growth. This in turn has the potential to feedback into economic development across both nations thereby reducing the gap between the urban rich and the rural poor. The speed and the extent to which this occurs will be determined by national governments and is outside the scope of this paper, but at least a well formed, globally integrated, indigenous HCI community will be able to enhance the quality of ICT systems that are accessible to the socially excluded. This must be the desire of all engaged in socially responsible IT development. 


\section{References}

1. Economist Intelligence Unit: The 2006 e-readiness Rankings. (Retrieved January 9th 2007) (2006), from http://www.eiu.com/site_info.asp?info_name=eiu_2006_e_readiness_ rankings

2. Prestowitz, C.: China-India Entente Shifts Global Balance. Yale Global Online. (Retrieved January 9th 2007) (2005), from http://yaleglobal.yale.edu/display.article?id=5578

3. Joshi, A.: Institutionalizing HCI - the Challenges in India. CSI Communications 29(3) (2005)

4. Rogers, E.M.: Diffusion of Innovation. Free Press, New York (1962)

5. Smith, A., Gulliksen, J., Bannon, L.: Building Usability in India: Reflections from the Indo European Systems Usability Partnership. In: McEwan, T., Gulliksen, J., Benyon, D. (eds.) People and Computers XIX - The Bigger Picture: Proceedings of HCI 2005, Springer, Heidelberg (2005)

6. Earthy, J.: Usability Maturity Model: Human Centeredness Scale. Lloyd's Register (1998)

7. Liu, Z., Zhang, L., Zhang, H.: An Organisational Human-centeredness Assessment at Chinese Software Enterprises. In: Proceedings of APCHI2002, pp. 251-259. Academic Press, London (2002)

8. NASSCOM: Why India? National Association of Software and Service Companies, (Retrieved January 9th 2007), from http://www.nasscom.in/Nasscom/templates/NormalPage.aspx?id=6316

9. Joshi, A.: HCI in SE Process Literature. Indo-Dan HCI Research Symposium, Guwahati (2006)

10. Smith, A., Yetim, F.: Global Human-computer Systems: Cultural Determinants of Usability. Special Issue Editorial. Interacting with Computers 16(1), 1-5 (2004)

11. Hoft, N.: Developing a Cultural Model. In: del Galdo, E., Nielsen, J. (eds.) International User Interfaces, pp. 41-73. Wiley Computer Publishing, New York (1996)

12. Hall, E.T.: Beyond Culture, Doubleday. Garden City, New York (1976)

13. Trompenaars, F.: Managing Across Cultures. Business Books. Random House Books, London (1993)

14. Victor, D.A.: International Business Communication. HarperCollins, New York (1992)

15. Hofstede, G.: Cultures and Organizations: Software of the Mind. Mc Graw-Hill, Berkshire, UK (1991)

16. Smith, A., Dunckley, L.: Using the LUCID Method to Optimize the Acceptability of Shared Interfaces. Interacting with Computers 9(3), 333-345 (1998)

17. McSweeney, B.: Hofstede's Model of National Cultural Differences and Consequences: a Triumph of Faith - a Failure of Analysis. Human Relations 55(1), 89-118 (2002)

18. Gulliksen, J., Göransson, B., Boivie, I., Persson, J., Blomkvist, S., Cajander, A., Cajander, A: Key principles for user centred systems design. Behaviour and Information Technology 22(6), 397-409 (2003)

19. Beyer, H., Holtzblatt, K.: Contextual Design: Defining Customer-centred Systems. Morgan Kaufmann, San Francisco (1998)

20. Dray, S.: Usable for the World: A Practical Guide to International User Studies. In: Day, D., Dunckley, L. (eds.) Designing for Global Markets 3. Proceedings of IWIPS 2001, IWIPS, pp. 153-154 (2001)

21. Murphy, J.: Modelling, Designer-tester-Subject Relationships in International Usability Testing. In: Day, D., Dunckley, L. (eds.) Designing for Global Markets 3. IWIPS 2001, IWIPS 2001, Milton Keynes, UK, pp. 33-44 (2001) 
22. Yeo, A.: Usability Evaluation in Malaysia. In: Proceedings of 4th Asia Pacific Computer Human Interaction Conference: APCHI 2000, pp. 275-280. Elsevier, Amsterdam (2000)

23. Yeo, A.: Global Software Development Lifecycle: an Exploratory Study. In: Jacko, J., Sears, A., Beaudouin-Lafon, M., Jacob, R. (eds.) CHI 2001: Conference on Human Factors in Computing Systems, pp. 104-111. ACM Press, New York (2001)

24. Chavan, A.: The Bollywood Method. In: Schaffer, E. (ed.) Institutionalization of Usability; a Step-by-step Guide, pp. 129-130. Adisson Wesley, New York (2004) 